\title{
Auto-encoders for Noise Reduction in Scanning Transmission Electron Microscopy
}

\author{
James P. Buban ${ }^{1}$ and Si-Young Choi ${ }^{1}$ \\ ${ }^{1}$ Korea Institute of Materials Science, 797 Changwondaero, Seongusan-gu, Changwon, Gyeongnam, \\ South Korea 51508
}

In recent years, machine learning has been applied to a wide variety of fields with increasing success. With the current availability of cheap computing power and the advent of deep learning, the applications of several powerful neural network architectures, such as convolutional neural networks, recurrent neural networks and auto-encoding neural networks, have shown remarkable accuracy in classifying various data sets [1]. In particular, the auto-encoder (AE) architecture excels at recovering signal from noisy, or corrupted input data [2]. In the scanning transmission electron microscope (STEM), images are often plagued by various sources of noise. This is especially problematic for imaging beam sensitive materials where the scan speed is exceptionally fast and/or the beam current is very low [3]. Here, we investigate the applicability of using AEs for noise reduction in STEM images.

The basic model for a neural network is an input layer, one or more hidden layers (for feature learning) and an output layer. The goal is to train the network to classify, or make predictions, in a generalized way so that the network can still accurately classify previously unseen data. For the basic auto-encoder, the network architecture consists of two main layers (figure 1). The first is an encoding layer which takes the input data and maps it to a lower dimensional (or compressed) "code", and the second is a decoding layer, which maps the compressed "code" to an output while attempting to recover the original input data. In this work, both the encoding and the decoding layers actually consist of multiple hidden layers, to create the so-called "stacked auto-encoder", which has the ability to learn more abstract features. In general, the network is trained under supervision using the back propagation method. Here, we minimize the $\mathrm{L}_{2}$-norm (difference squared) cost function and back propagate the resulting error.

Figure 2 shows the results of independent test data after training an AE on a lattice of Gaussian functions with a fixed width and alternating intensities of 1.0 and 0.7 with respect to the image maximum in order to reproduce the intensities found in Z-contrast images of $\mathrm{SrTiO}_{3}$. The network was trained on 700,000 sample images of $28 \times 28$ pixels, each of which was randomly translated, then rotated between $0^{\circ}$ and $45^{\circ}$, and finally uniform noise was added with a signal-to-noise ratio of 0.33 before the samples were used as input to the auto-encoder. For this example, the output of the AE recovers the uncorrupted image with an average error of approximately $10 \%$ per pixel on an independent test set.

To apply these results to a real image, we use a simple floating window technique to take advantage of the oversampling in STEM images. A series of 28x28 pixel windows are extracted from a STEM image, which are individually processed by the trained $\mathrm{AE}$ and then recombined to give a reconstructed image. Figure 3 shows the results of the floating window process with a step size of 2 pixels for a fast-scan annular dark field STEM image of [001] $\mathrm{SrTiO}_{3}$ that includes a Ruddlesden-Popper defect. In this reconstruction, the much of the noise has been removed and the positions of the Sr-columns are easily discernable. Although the AE was trained on a perfect lattice of a fixed lattice parameter, one finds that in the reconstructed image, local variations of the lattice constant as well as lattice defects are easily detected. Improvements in the AE network training should increase the accuracy of the TiO-column positions and as well as improve the contrast variation in the processed image. 


\section{References:}

[1] Y LeCun et al, Nature 521 (2015) p. 436.

[2] P Vincent et al, Proceeding of the $25^{\text {th }}$ International Conference on Machine Learning (2008) p.1096.

[3] J Buban et al, Journal of Electron Microscopy 59 (2009), p. 103.

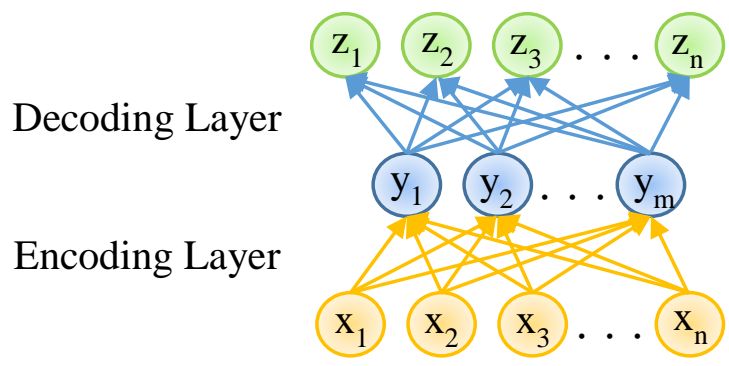

Figure 1: A schematic for the auto-encoder architecture. The input, compressed and output data sets are represented by the vectors $\left(x_{1}, \ldots, x_{n}\right),\left(y_{1}, \ldots, y_{m}\right)$, and $\left(z_{1}, \ldots, z_{n}\right)$, respectively, with $m<n$.

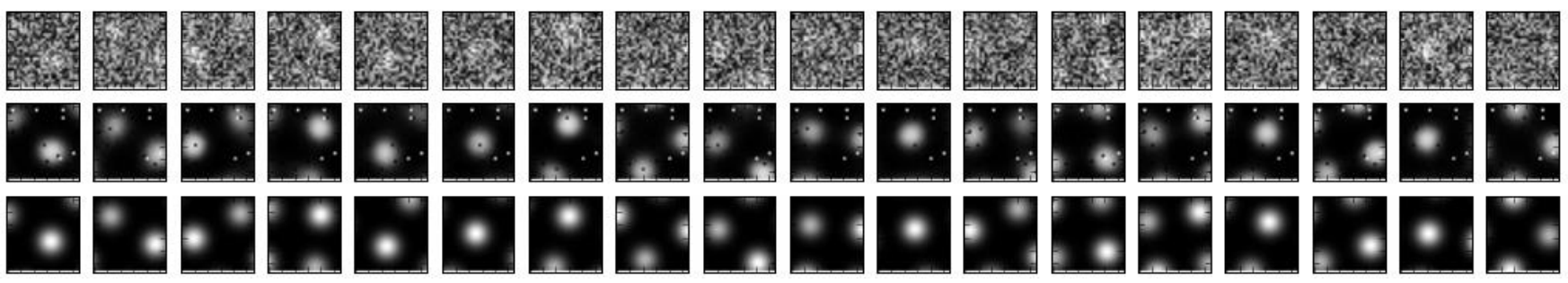

Figure 2. A series of test data sampled independently of the training set. Examples of the input images corrupted by noise, corresponding output (predicted image) from the auto-encoder described above, and the correct images (before noise corruption) are displayed in the first, second and third rows, respectively. The back propagation method trains the network while minimizing the error of the squared difference in pixel intensity between the outputs (second row) and the correct images (third row).
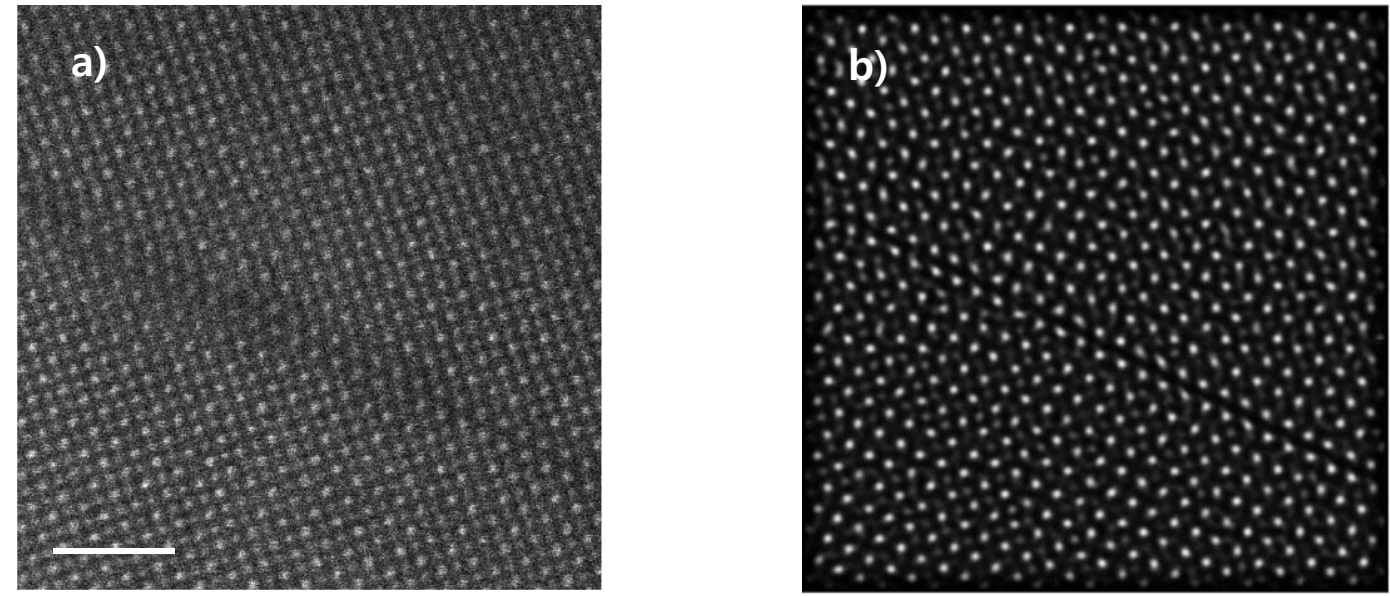

Figure 3. a) The raw fast-scan STEM image with a pixel dwell time of 2 microseconds. b) The reconstructed image using a window step size of 3 pixels with windows processed using the AE implemented in figure 2. Note that local variations in the unit cell, namely elongation (perpendicular to the defect plane) near the defect are reproduced in the processed image. 